\title{
Ground Truth for Diffusion MRI in Cancer: A Model-Based Investigation of a Novel Tissue-Mimetic Material
}

DOI:

10.1007/978-3-319-19992-4_14

Document Version

Accepted author manuscript

Link to publication record in Manchester Research Explorer

\section{Citation for published version (APA):}

Mchugh, D., Zhou, F., Cristinacce, P., Naish, J. H., \& Parker, G. (2015). Ground Truth for Diffusion MRI in Cancer: A Model-Based Investigation of a Novel Tissue-Mimetic Material. In International Conference on Information Processing in Medical Imaging: IPMI 2015: Information Processing in Medical Imaging pp 179-190 (Vol. 9123, pp. 179-190). (Lecture Notes in Computer Science (LNCS); Vol. 9123). Springer Nature. https://doi.org/10.1007/978-3319-19992-4_14

Published in:

International Conference on Information Processing in Medical Imaging

\section{Citing this paper}

Please note that where the full-text provided on Manchester Research Explorer is the Author Accepted Manuscript or Proof version this may differ from the final Published version. If citing, it is advised that you check and use the publisher's definitive version.

\section{General rights}

Copyright and moral rights for the publications made accessible in the Research Explorer are retained by the authors and/or other copyright owners and it is a condition of accessing publications that users recognise and abide by the legal requirements associated with these rights.

\section{Takedown policy}

If you believe that this document breaches copyright please refer to the University of Manchester's Takedown Procedures [http://man.ac.uk/04Y6Bo] or contact uml.scholarlycommunications@manchester.ac.uk providing relevant details, so we can investigate your claim.

\section{OPEN ACCESS}




\title{
Ground Truth for Diffusion MRI in Cancer: A Model-Based Investigation of a Novel Tissue-Mimetic Material
}

\author{
Damien J. McHugh ${ }^{1,2,3}$, Fenglei Zhou ${ }^{1,3,4}$, Penny L. Hubbard Cristinacce ${ }^{1,2}$, \\ Josephine H. Naish ${ }^{1,2}$, and Geoffrey J.M. Parker ${ }^{1,2,3}$ \\ 1 Centre for Imaging Sciences, The University of Manchester, Manchester, UK \\ 2 Biomedical Imaging Institute, The University of Manchester, Manchester, UK \\ ${ }^{3}$ CRUK and EPSRC Cancer Imaging Centre in Cambridge and Manchester, UK \\ 4 Materials Science Centre, The University of Manchester, Manchester, UK
}

\begin{abstract}
This work presents preliminary results on the development, characterisation, and use of a novel physical phantom designed as a simple mimic of tumour cellular structure, for diffusion-weighted magnetic resonance imaging (DW-MRI) applications. The phantom consists of a collection of roughly spherical, micron-sized core-shell polymer 'cells', providing a system whose ground truth microstructural properties can be determined and compared with those obtained from modelling the DWMRI signal. A two-compartment analytic model combining restricted diffusion inside a sphere with hindered extracellular diffusion was initially investigated through Monte Carlo diffusion simulations, allowing a comparison between analytic and simulated signals. The model was then fitted to DW-MRI data acquired from the phantom over a range of gradient strengths and diffusion times, yielding estimates of 'cell' size, intracellular volume fraction and the free diffusion coefficient. An initial assessment of the accuracy and precision of these estimates is provided, using independent scanning electron microscope measurements and bootstrap-style simulations. Such phantoms may be useful for testing microstructural models relevant to the characterisation of tumour tissue.
\end{abstract}

Keywords: tumour microstructure - diffusion MRI - biomimetic phantoms $\cdot$ core-shell microspheres $\cdot$ coaxial electrospraying

The final publication is available at Springer via http://dx.doi.org/10.1007/9783-319-19992-4_14

\section{Introduction}

The dependence of the DW-MRI signal on tissue microstructural properties underpins the use of DW-MRI in investigating cellular changes in a variety of pathological conditions, such as stroke [1] and cancer [2]. While the apparent diffusion coefficient (ADC) derived from DW-MRI can provide sensitivity to 
microstructural changes, it lacks specificity as it is potentially affected by a range of tissue properties without directly characterising them. This has motivated the use of microstructural models that describe the DW-MRI signal as a function of sequence parameters and specific tissue properties. Such models typically represent tissue in terms of intra- and extra-cellular compartments, with cells modelled as idealised shapes with impermeable or permeable membranes. These models can be fitted to acquired data to estimate parameters such as cell size, compartment volume fractions and diffusivities. For example, optic nerve has been modelled as a combination of ellipsoids (axons), spheres (glial cells), and an extracellular space, with a multi-compartment exchange model used to estimate the size, volume fraction, diffusivities and permeabilities of the compartments [3]. Axons have also been modelled as impermeable cylinders with a distribution of diameters, enabling axon size distributions to be estimated in fixed tissue [4] and in vivo [5]. A model comprising monodisperse impermeable cylinders has also been used to estimate indices of axonal diameter and density in human brains [6]. Recently, impermeable spheres have been used to model tumour tissue, allowing cell size and volume fractions to be estimated in vivo [7]. The increasing interest in using such model-based analyses to extract specific microstructural information from DW-MRI data motivates the development of model systems where the ground truth can be independently determined and used to validate microstructural models.

Such model systems range from in silico geometries $[8,9]$ and synthetic physical phantoms $[10,11,12]$, through to plant [13] and fixed biological tissue [3,4]. Synthetic physical phantoms provide a system where ground truth properties can potentially be characterised and varied more easily than with biological tissue, but less easily than in silico models, and where the experimental methods more closely reflect those used when investigating real tissue than when using in silico approaches. A number of studies have used synthetic phantoms to test DW-MRI methods developed for applications in white matter. Fibre phantoms with variable packing density have been used to study extracellular diffusion [10], while silica microcapillaries have been used to mimic intra-axonal diffusion and test microstructural modelling methods [12]. Recently, coaxial electrospinning has been used to develop axon-mimicking hollow fibres whose orientation and size can be controlled [14,15], with intracellular diffusivity measurements showing sensitivity to fibre size [11]. In contrast to these white matter phantoms that seek to mimic specific microstructural properties, phantom studies in oncology have focussed on free-diffusion phantoms for validation of ADC values. For example, ice-water phantoms have been investigated as a way of validating ADC measurements in multi-centre clinical trials [16]. While such phantoms are useful for assessing the repeatability and reproducibility of ADC measurements, they are not suitable for studying the specific tissue properties that underlie tumour ADC measurements.

The present work describes in silico and experimental investigations undertaken to validate an analytic model of the DW-MRI signal relevant to the characterisation of tumour tissue. This model was initially investigated through Monte 
Carlo diffusion simulations, allowing a comparison between analytic and simulated signals. The model was then applied to experimental DW-MRI data obtained from a novel physical phantom designed as a simple mimic of tumour tissue. The phantom was developed using coaxial electrospraying [17] to generate a collection of roughly spherical, micron-sized hollow polymer particles, mimicking cells. Scanning electron microscope (SEM) imaging was used to obtain a ground truth measurement of the mean sphere size, which was compared with the size estimated from modelling the DW-MRI data. The paper starts with a description of the model and the Monte Carlo simulations used for in silico validation. Details of the phantom construction and characterisation are then presented, followed by a description of the MR experiments and the results.

\section{Microstructural Model}

The microstructural model considered here consists of diffusion in two nonexchanging compartments: a restricted intracellular compartment with volume fraction $f_{i}$ and diffusion coefficient $D_{i}$, and a hindered extracellular compartment with volume fraction $f_{e}=1-f_{i}$ and diffusion coefficient $D_{e}$. The DW-MRI signal, $S$, is given by

$$
S=f_{i} S_{i}+\left(1-f_{i}\right) S_{e},
$$

where

$$
\begin{gathered}
S_{i}=\exp \left(-2 \gamma^{2} G^{2} \sum_{m=1}^{\infty} \frac{1}{\alpha_{m}^{2}\left(\alpha_{m}^{2} R^{2}-2\right)}\left[\frac{2 \delta}{\alpha_{m}^{2} D_{i}}\right.\right. \\
\left.\left.+\frac{2 e^{-\alpha_{m}^{2} D_{i} \delta}+2 e^{-\alpha_{m}^{2} D_{i} \Delta}-e^{-\alpha_{m}^{2} D_{i}(\Delta-\delta)}-e^{-\alpha_{m}^{2} D_{i}(\Delta+\delta)}-2}{\alpha_{m}^{4} D_{i}^{2}}\right]\right), \\
S_{e}=\exp \left(-\gamma^{2} \delta^{2} G^{2}(\Delta-\delta / 3) \frac{D_{e}}{1+f_{i} / 2}\right) .
\end{gathered}
$$

Equation (2) is the pulsed gradient spin-echo (PGSE) signal for diffusion restricted inside an impermeable sphere of radius $R$ [18] (assuming a Gaussian phase distribution [19]), and (3) gives the signal for hindered extracellular diffusion with the diffusion coefficient reduced by a tortuosity factor, $1+f_{i} / 2[20]$. The $\alpha_{m}$ in (2) is obtained from the $m$ th root of $\alpha_{m} R J_{3 / 2}^{\prime}\left(\alpha_{m} R\right)-\frac{1}{2} J_{3 / 2}\left(\alpha_{m} R\right)=0$, where $J_{3 / 2}$ is the Bessel function of the first kind, order $3 / 2$ [18,19]. The signals calculated analytically from (1)-(3) were compared with synthetic signals generated from Monte Carlo diffusion simulations. The Camino toolkit [8] was used to perform random walk simulations in a 3D geometry of monodisperse, packed impermeable spheres. All simulations had spatial and temporal resolutions of $0.655 \mathrm{~mm}$ and $0.0357 \mathrm{~ms}$, respectively, used 195000 walkers and had intra- and extra-cellular diffusion coefficients set to $2 \times 10^{-3} \mathrm{~mm}^{2} / \mathrm{s}$. Models with different cell sizes $(R=7-30 \mu \mathrm{m})$ and intracellular volume fractions $\left(f_{i}=0.16-0.71\right)$ were used in separate simulations. For each combination of $R$ and $f_{i}$, synthetic 
signals were generated for PGSE sequences with a range of gradient strengths, $G=0-263 \mathrm{mT} / \mathrm{m}$, and separations, $\Delta=12-45 \mathrm{~ms}$, with the gradient duration $\delta=4 \mathrm{~ms}$. These sequence parameters can be obtained on preclinical scanners, and match the range used in the phantom MR experiments (see below). Figure 1 shows an example of the geometry used in these simulations, and plots synthetic (circles) and analytic (dashed lines) signals as a function of $G$ and $\Delta$ for different combinations of $R$ and $f_{i}$. In general, good agreement was found between the synthetic and analytic signals over a range of tissue properties and sequence parameters, with a maximum difference between simulated and analytic signals of 0.02 . This suggests that (1)-(3) provide an accurate description of this simple tissue model.

\section{Phantom Construction and Characterisation}

Coaxial electrospraying was performed using polyethylene glycol (PEG) dissolved in chloroform for the core, and polycaprolactone (PCL) dissolved in chloroform for the shell. The PEG solution was injected into the inner needle of a coaxial spinneret at a flow rate of $1 \mathrm{ml} / \mathrm{h}$, while the PCL solution was injected into the outer needle at $3 \mathrm{ml} / \mathrm{h}$. A voltage of $9 \mathrm{kV}$ was applied between the spinneret and a ground electrode (a thin aluminium plate) placed $20 \mathrm{~cm}$ below.
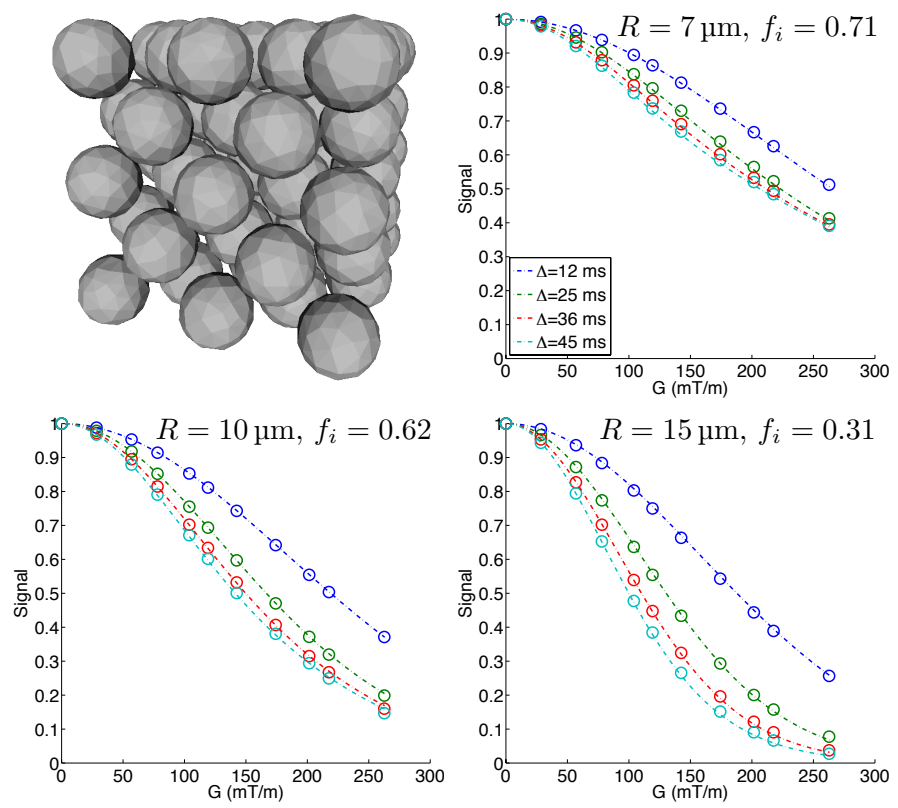

Fig. 1: Spherical cell geometry used in the simulations, along with synthetic (circles) and analytic (dashed lines) signals plotted as a function of $G$ and $\Delta$ for different combinations of $R$ and $f_{i}$. 
The core-shell spheres, which form as the liquid jet emerging from the spinneret breaks up, were collected on a copper wire placed on the ground electrode, forming a bulk sample over a period of $1-2 \mathrm{~h}$. The wire was then removed, leaving a bulk sample structured as a hollow cylinder. Two parts of the bulk phantom were used for SEM and MR characterisation, respectively. For the MR experiments the phantom was placed in an NMR tube ( $5 \mathrm{~mm}$ outer diameter), which was filled with cyclohexane approximately one week before scanning. Cyclohexane provides the MR signal in these experiments and was chosen instead of water as PCL is hydrophobic and therefore prevents water molecules reaching the intracellular space. The potential for using other polymers is currently being investigated, which may allow water to be used instead of cyclohexane.

From the part of the bulk sample set aside for SEM analysis, ten subsamples were taken and imaged with a Phenom G2 Pro desktop SEM. These ten SEM images were then used to estimate the size of the spheres. Analysis was carried out using ImageJ (National Institutes of Health, Bethesda, Maryland, USA, http://imagej.nih.gov/ij/), and began by selecting which spheres in each image would be measured. In order to avoid potential bias in choosing spheres manually, a grid was placed on each SEM image, and spheres which contained a grid intersection were chosen as potential candidates for measurement. Not all of these spheres could be measured, however, as spheres in the foreground could obstruct those behind. As such, candidate spheres were selected for measurement if two perpendicular lines could be drawn from one edge to another, approximately through the sphere centre, without other spheres occluding the edges. In some cases the edges merged with another sphere, making the boundary less well defined. In this way, two diameter measurements were made for each chosen sphere, with the final estimate taken as the mean of these two lengths. At least ten spheres per image were chosen for measurement, and the process was performed independently by two observers after deciding which spheres were to be analysed. The mean difference and limits of agreement were calculated to compare the two observers' measurements [21]. For consistency with the MR analysis, the measurements are reported as sphere radii, as opposed to diameters. It should be noted that these measurements provide an estimate of the outer radius of the spheres, which is larger than the internal radius due to the non-zero thickness of the sphere walls.

\section{MR Methods}

\subsection{MR Acquisition}

MR experiments were carried out on $7 \mathrm{~T}$ Bruker system (Bruker BioSpin, Ettlingen, Germany), using a transmit/receive volume coil. Data were acquired using four separate PGSE sequences, each with a different gradient separation: $\Delta=12,25,36,45 \mathrm{~ms}$. For each $\Delta$ scan, images were acquired at seven gradient strengths: $G=0,28.5,78.1,119,147,202,263 \mathrm{mT} / \mathrm{m} ; \delta$ was fixed at $4 \mathrm{~ms}$ for each scan. In order to maximise signal to noise ratio (SNR), the lowest possible echo time (TE) was chosen for each $\Delta$, giving $\mathrm{TE}=21.2,34.2,45.2,54.2 \mathrm{~ms}$ for 
$\Delta=12,25,36,45 \mathrm{~ms}$, respectively. Each scan acquired images with three diffusion gradient directions, using a spin-echo readout with a $30 \mathrm{~mm} \times 30 \mathrm{~mm}$ field of view, $128 \times 128$ matrix, $1 \mathrm{~mm}$ slice thickness and a $2500 \mathrm{~ms}$ repetition time.

\subsection{MR Analysis}

The phantom ROI was defined by thresholding the $G=0 \mathrm{mT} / \mathrm{m}$ images to leave only the voxels within the phantom, excluding those containing the free cyclohexane. The equivalent voxels in each diffusion-weighted image were found, and the mean signal intensity taken for each $G$ value to boost SNR. Repeating this for each $\Delta$ scan therefore gave ROI-averaged signal intensities as a function of $G$ and $\Delta$, which were then normalised to the unweighted signal for their respective $\Delta$ scans. The two-compartment analytic expression given by (1)-(3) was then fitted to these normalised signals. The free diffusivities in the intra- and extra-cellular spaces were assumed to be equal, $D_{i}=D_{e}=D$. Two methods of performing the fitting were investigated. First, the full four-parameter fit was carried out, yielding estimates of each model parameter (cell radius, $R$, intracellular volume fraction, $f_{i}$, free diffusivity, $D$, and unweighted signal, $S_{0}$ ) directly from the ROI-averaged phantom signals. Second, $D$ was fixed during the optimisation, with the fit returning estimates of $R, f_{i}$ and $S_{0}$ only. In this case, $D$ was fixed at the value of the diffusion coefficient measured in the free cyclohexane, which serves as a ground truth measurement of the free diffusion coefficient. This value was obtained by averaging the median ADC values from a ROI in the free cyclohexane, over the different $\Delta$ experiments and gradient directions. The ADC fits were performed on a voxel-wise basis using maximum likelihood (ML) fitting [22], with the full range of $G$ for each $\Delta$ experiment. The ML method used a single Rician probability density function (PDF) in the objective function and was appropriate here as the signals used in the ADC fitting were not averaged [23].

For the microstructural estimates, the fitting procedure was repeated for a range of starting values, and the final result taken as the fit which gave the lowest value of the objective function. Three starting values were picked at random for each parameter, within a wide range of possible values: $R=1-35 \mu \mathrm{m}, f_{i}=0-1$, $D=0.1-3.1 \times 10^{-3} \mathrm{~mm}^{2} / \mathrm{s}$. The fitting was then repeated for each combination of these randomly chosen values, giving $3^{3}=27$ different fits; only one starting value was used for $S_{0}$, which was 1 . Picking $2,3,4,5$ or 6 starting values for each parameter (giving $8,27,64,125$ and 216 repeated fits, respectively) was observed to have negligible effect on the final results. Different random selections within the same range also had negligible effect on the results, suggesting that the values of the fitted parameters were not an artefact of the starting values. Apart from fixing $D$ in the second fitting method, no constraints were applied to any of the fitted parameters. In contrast to the non-averaged signals used for the free cyclohexane ADC calculations, the fits to the two-compartment model used averaged signals, making the use of a single Rician PDF for ML fitting no longer appropriate [23]. As the PDF for averaged Rician-distributed signals has 
no closed-form expression, the ML method used above cannot be employed, and least squares (LS) fitting was used instead.

Bootstrap-style simulations were performed to investigate the precision of the parameter estimates. Specifically, propagation of errors was used to calculate the errors on the normalised signals from the phantom ROI, according to:

$$
\sigma^{\prime}\left(G_{i}, \Delta\right)=S^{\prime}\left(G_{i}, \Delta\right) \sqrt{\left(\frac{\sigma\left(G_{i}, \Delta\right)}{S\left(G_{i}, \Delta\right)}\right)^{2}+\left(\frac{\sigma\left(G_{1}, \Delta\right)}{S\left(G_{1}, \Delta\right)}\right)^{2}},
$$

where $\sigma^{\prime}\left(G_{i}, \Delta\right)$ is the standard deviation (SD) of the normalised signal for the $i$ th gradient strength, $S^{\prime}\left(G_{i}, \Delta\right)$ is the normalised mean signal for the $i$ th gradient strength, $\sigma\left(G_{i}, \Delta\right)$ and $S\left(G_{i}, \Delta\right)$ are the SD and mean of the unnormalised $i$ th gradient strength ROI-averaged signal and $\sigma\left(G_{1}, \Delta\right)$ and $S\left(G_{1}, \Delta\right)$ are the SD and mean of the unnormalised $G=0 \mathrm{mT} / \mathrm{m}$ ROI-averaged signal. The $\sigma^{\prime}\left(G_{i}, \Delta\right)$ values were then used to construct $95 \%$ confidence intervals, $c i$, for each $S^{\prime}\left(G_{i}, \Delta\right)$. A new set of signals, $S_{\text {new }}^{\prime}(G, \Delta)$, was generated by adding or subtracting a random amount from $S^{\prime}(G, \Delta)$, such that $95 \%$ of $S_{\text {new }}^{\prime}(G, \Delta)$ lay within $S^{\prime}(G, \Delta) \pm c i$. Parameter estimates from the new set of signals were then obtained using the fitting described above, and this process was repeated for 10000 sets of synthetic signals. Histograms of the resulting parameter estimates were then plotted. All model fitting was performed using the Nelder-Mead simplex algorithm in MATLAB 2010a (The MathWorks, Natick, MA, USA).

\section{Results and Discussion}

\subsection{Microstructure Characterisation}

An example SEM image is shown in Fig. 2a, illustrating the grid placement and labelling of the spheres chosen for measurement. There is a tendency for the spheres to merge together, indicating that the bulk phantom is not simply a collection of individual spheres. Also note the presence of one much smaller sphere, with a radius of approximately $1.8 \mu \mathrm{m}$; this is visible in Fig. 2a (labelled with the number 8 and indicated by the white arrow), along with three other similarly sized spheres. While such spheres broaden the size distribution, there appear to be few of them and their low volume fraction means they would be expected to contribute little to the measured signal. Diameter measurements were made for a total of 160 spheres. The histogram of the corresponding radii measured by one observer is shown in Fig. 2b. There was good agreement between the measurements made by the two observers; mean difference between the radii estimates: $0.01 \mu \mathrm{m}$, with $95 \%$ limits of agreement: $-0.5 \mu \mathrm{m}$ to $0.6 \mu \mathrm{m}$, and $95 \%$ confidence interval for the mean difference: $-0.03 \mu \mathrm{m}$ to $0.06 \mu \mathrm{m}$. Both observers' measurements had a mean $\pm \mathrm{SD}$ of $(7.9 \pm 1.1) \mu \mathrm{m}$, which was taken as the ground truth outer radius. 

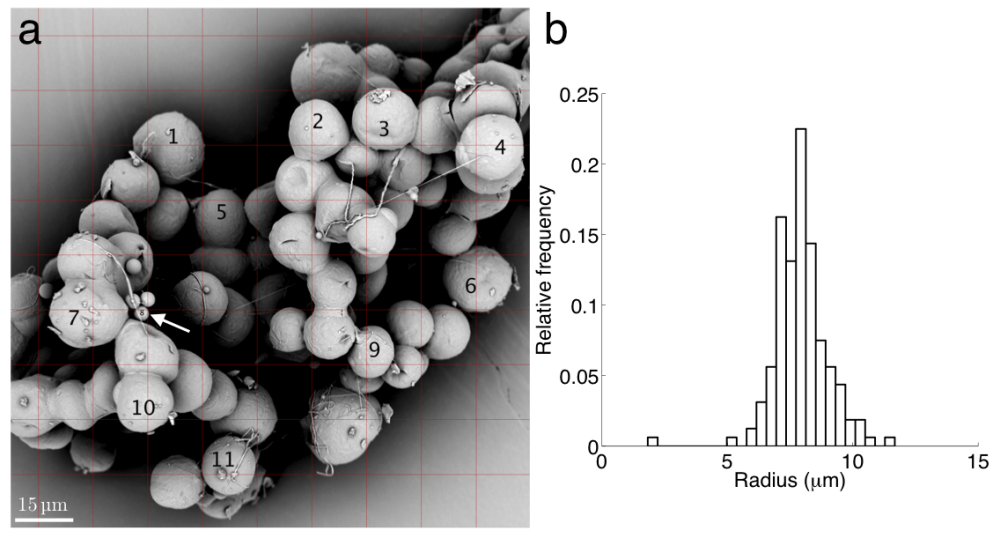

Fig. 2: (a) One of the ten SEM images used for the sphere size measurements, illustrating grid placement and the spheres chosen to measure. (b) Histogram of 160 radii measurements made by one observer; mean $\pm \mathrm{SD}=(7.9 \pm 1.1) \mu \mathrm{m}$.

\subsection{MR Experiments}

Figure 3a shows the $G=0 \mathrm{mT} / \mathrm{m}, \Delta=12 \mathrm{~ms}$ image, where the red circle defines the free cyclohexane ROI, and the lower-signal annulus corresponds to the phantom region. The free cyclohexane ADC results are shown in Fig. 3b, where the cyclohexane $\mathrm{ADC}$ is shown to be independent of $\Delta$ and direction, as expected for fluid undergoing free diffusion. The mean $\pm \mathrm{SD}$ ADC over all $\Delta$ and directions was $(1.36 \pm 0.03) \times 10^{-3} \mathrm{~mm}^{2} / \mathrm{s} ; D$ was therefore fixed to this value when fitting to the phantom data using the second method described above. At high $G$ and high $\Delta$ the phantom signal was almost completely attenuated, resulting in very low SNR. For the ROI-averaged phantom signals, the highest $G$ for $\Delta=36 \mathrm{~ms}$, and the two highest $G$ for $\Delta=45 \mathrm{~ms}$ gave SNR $<2$ and were excluded from the fitting. The fits to the model are shown in Figs. 3c ( $D$ estimated) and $3 \mathrm{~d}$ ( $D$ fixed). When $D$ was an estimated parameter the fit gave $R=6.1 \mu \mathrm{m}, f_{i}=0.23, D=1.40 \times 10^{-3} \mathrm{~mm}^{2} / \mathrm{s}$ and $S_{0}=0.994$. When $D$ was fixed at $1.36 \times 10^{-3} \mathrm{~mm}^{2} / \mathrm{s}$ the estimates were $R=6.1 \mu \mathrm{m}, f_{i}=0.22$ and $S_{0}=0.993$. The fitted value of $D$ was therefore only $3 \%$ higher than that obtained from the independent measurement, and fixing $D$ was observed to have little impact on the estimates of $R, f_{i}$, and $S_{0}$.

Figure 4 shows the histograms obtained from the bootstrap-style simulations used to investigate the precision of the microstructural estimates obtained from the phantom data. The confidence intervals that were used in these simulations (derived using (4)) ranged from 0.01 to 0.06. The top row shows the results when all four model parameters were estimated, and the bottom row shows the results obtained when $D$ was fixed. Overall, fixing $D$ was found to have little effect on the mean and SD of $R, f_{i}$, and $S_{0}$ estimates. When $D$ was a fitted parameter, 

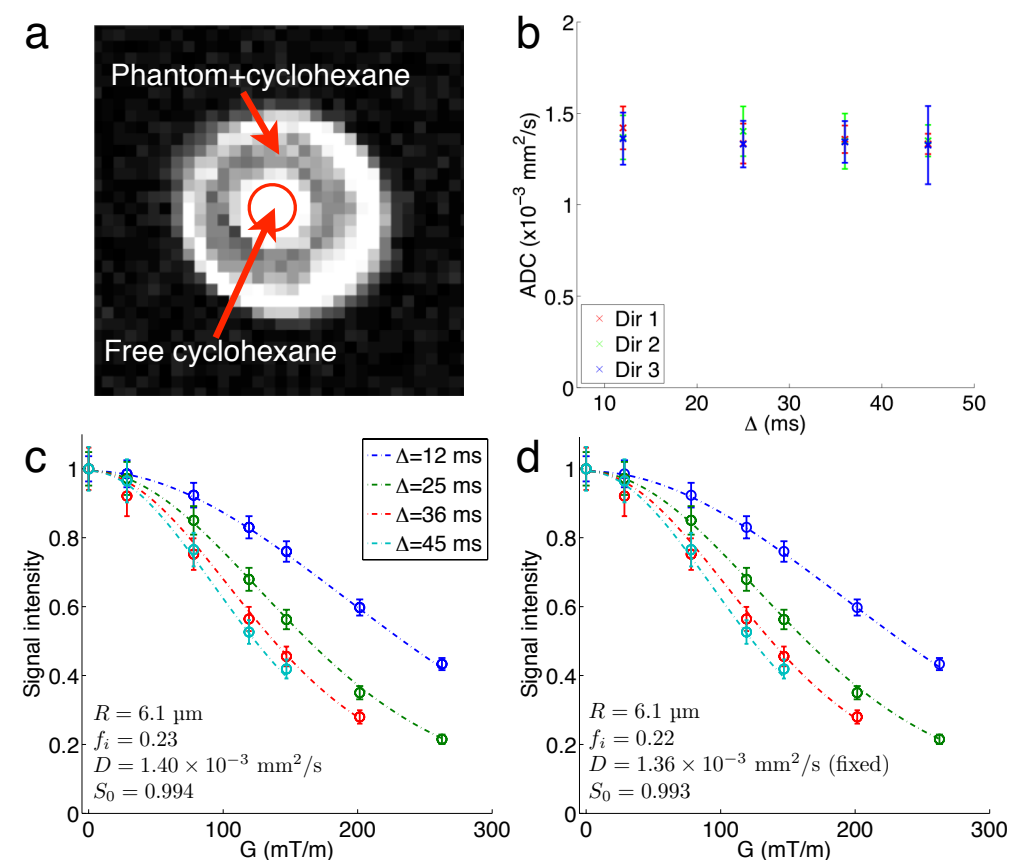

Fig. 3: (a) $G=0 \mathrm{mT} / \mathrm{m}, \Delta=12 \mathrm{~ms}$ image; the red circle defines the free cyclohexane ROI, and the lower-signal annulus corresponds to the phantom region. (b) Free cyclohexane ADC as a function of $\Delta$. (c) Signals (circles) and model fitting (dashed lines) as a function of $G$ and $\Delta$; error bars correspond to the $95 \%$ ci as described. (d) As in (c) but with $D$ fixed.

the mean $\pm \mathrm{SD}$ over the 10000 fits were $R=(6.2 \pm 0.9) \mu \mathrm{m}, f_{i}=0.24 \pm 0.03$, $D=(1.40 \pm 0.09) \times 10^{-3} \mathrm{~mm}^{2} / \mathrm{s}$, and $S_{0}=0.994 \pm 0.009$. When $D$ was fixed, the values were $R=(6 \pm 1) \mu \mathrm{m}, f_{i}=0.22 \pm 0.03$, and $S_{0}=0.993 \pm 0.008$. Taking the SD of these distributions as a measure of the precision of the parameter estimates, these results suggest that fixing $D$ to the free fluid value has little impact on the precision of the cell size or volume fraction estimated from the phantom data. This also suggests that the diffusivity measured for the free cyclohexane provides a good estimate of the unhindered diffusivity in the phantom. The radius histograms in Fig. 4 also indicate the mean radius determined from SEM, $7.9 \mu \mathrm{m}$ (red lines). The standard error on this estimate is $\approx 0.09 \mu \mathrm{m}$; an error bar showing this would be approximately the same width as the red line so has been omitted. The precision of the two size estimates suggests that $R$ is lower than the outer sphere size measured using SEM. Qualitatively, this is expected given the non-zero thickness of the sphere wall, though measurements of this thickness are needed to see if this alone accounts for the difference. Modelling assumptions, sphere manufacturing imperfections and bias due to the use 

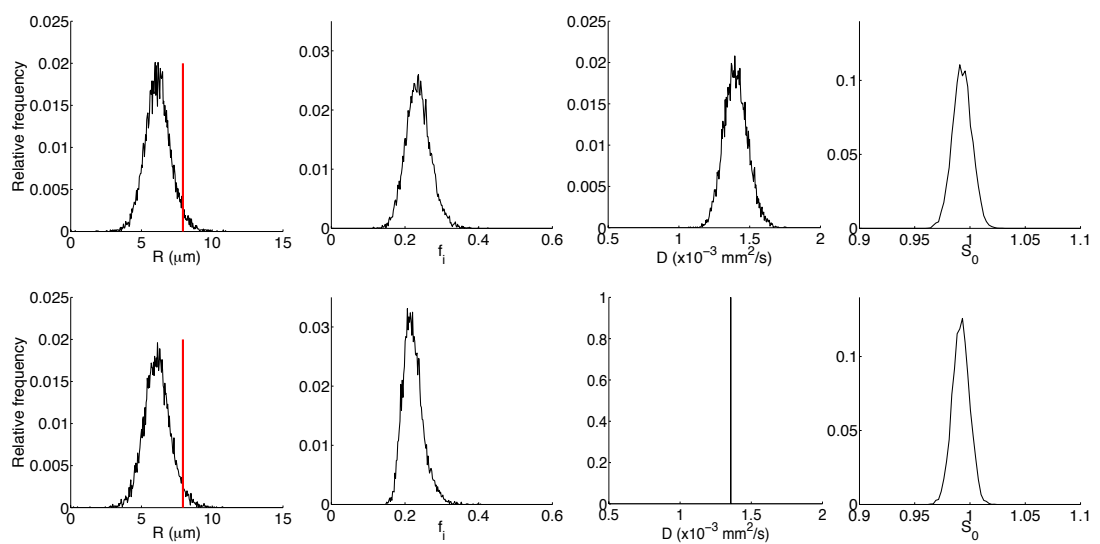

Fig. 4: Model parameter histograms from bootstrap-style simulations, when $D$ is estimated (top) and fixed (bottom). The red line on radius histograms indicates the mean radius obtained from SEM.

of LS fitting may also contribute to the difference. The estimated free diffusivity is consistent with the free cyclohexane ADC, and the volume fraction is plausible. Work to establish a ground truth volume fraction using high-resolution computed tomography is ongoing, which will allow the accuracy of the $f_{i}$ estimate to be assessed. The close agreement between the model estimates and ground truth observations for $R$ and $D$, combined with the very close agreement between theory and Monte Carlo simulations (Fig. 1), provides strong evidence in support of the use of the analytic model in (1)-(3) for assessing micron-scale spherical structures using DW-MRI.

The work presented here has focussed on one phantom, but it is clearly desirable to test the model in a range of phantoms with different microstructural characteristics. By altering parameters in the electrospraying process such as the electric field strength (applied voltage/working distance) and the flow rates of the polymer solutions, the inner radius of the resultant spheres, and the shell thickness, can be controlled. This approach has been used previously to generate electrospun fibre phantoms with different diameters [11], [15]. Typically, the electrospraying technique is capable of producing spheres in the micron and sub-micron size range, and we envisage being able to produce spheres over a physiologically-relevant size range. For example, a previous electrospraying study generated spheres with diameters ranging from $0.6-36 \mu \mathrm{m}$, although these were not hollow [24]. The bulk size of the phantom is mainly dependent on the sphere collecting efficiency and the length of time over which the electrospraying process is carried out. For example, the phantom used in this study had a length of $\sim 9 \mathrm{~mm}$ and an inner/outer annular radius of $\sim 0.7 \mathrm{~mm} / 1.3 \mathrm{~mm}$, and was produced over a period of 1-2 h. While we do not currently have data regarding the reproducibility of the sphere phantom construction process, based on previous 
experience of generating fibre phantoms with co-electrospinning, we expect the reproducibility to be reasonably good.

Finally, it should be noted that the phantom investigated here is clearly a vast oversimplification of actual tumour tissue, lacking intra- and extra-cellular structures, such as cell nuclei and collagen, respectively, as well as lacking a vascular component. The simplicity of the phantom is intended to facilitate the validation of a simple mathematical model, which is not designed to capture all aspects of tumour microstructure. Future work will look at applying the model to progressively more complex systems, such as modified phantoms and in vitro and in vivo tumour models, with the aim of developing an understanding of which aspects of tumour microstructure the DW-MRI signal is sensitive to.

\section{Conclusions}

This work described in silico and experimental validation of a biophysical model of the DW-MRI signal. Good agreement was found between Monte Carlo simulations and the analytic model, which was then used to obtain microstructural estimates from a novel physical phantom. While further characterisation of the phantom is needed to fully assess the accuracy of the DW-MRI estimates, the difference between the MR and SEM radii is qualitatively consistent with the non-zero thickness of the sphere walls. The estimated free diffusivity is consistent with the free cyclohexane ADC, and the estimated volume fraction is plausible. These phantoms may prove useful for evaluating the accuracy and precision of estimates from models relevant to the characterisation of tumour tissue.

Acknowledgements. The authors thank Matt Hall for assistance with the simulations, and acknowledge the assistance given by IT Services and the use of the Computational Shared Facility at The University of Manchester. This work was supported by the MRC and AstraZeneca, and used facilities funded by the BBSRC. This work was supported by CRUK [C8742/A18097]. This is a contribution from the Cancer Imaging Centre in Cambridge and Manchester, which is funded by the EPSRC and Cancer Research UK.

\section{References}

1. Roberts, T.P.L., Rowley, H.A.: Diffusion weighted magnetic resonance imaging in stroke. Eur. J. Radiol. 45, 185-194 (2003)

2. Padhani, A.R., Liu, G., Koh, D.M., Chenevert, T.L., Thoeny, H.C., Takahara, T., Dzik-Jurasz, A., Ross, B.D., Van Cauteren, M., Collins, D., Hammoud, D.A., Rustin, G.J.S., Taouli, B., Choyke, P.L.: Diffusion-weighted magnetic resonance imaging as a cancer biomarker: consensus and recommendations. Neoplasia 11, $102-125(2009)$

3. Stanisz, G.J., Szafer, A., Wright, G.A., Henkelman, R.M.: An analytical model of restricted diffusion in bovine optic nerve. Magn. Reson. Med. 37, 103-111 (1997) 
4. Assaf, Y., Blumenfeld-Katzir, T., Yovel, Y., Basser, P.J.: AxCaliber: a method for measuring axon diameter distribution from diffusion MRI. Magn. Reson. Med. 59, 1347-1354 (2008)

5. Barazany, D., Basser, P.J., Assaf, Y.: In vivo measurement of axon diameter distribution in the corpus callosum of rat brain. Brain 132, 1210-1220 (2009)

6. Alexander, D.C., Hubbard, P.L., Hall, M.G., Moore, E.A., Ptito, M., Parker, G.J.M., Dyrby, T.B.: Orientationally invariant indices of axon diameter and density from diffusion MRI. NeuroImage 52, 1374-1389 (2010)

7. Panagiotaki, E., Walker-Samuel, S., Siow, B., Johnson, S.P., Rajkumar, V., Pedley, R.B., Lythgoe, M.F., Alexander, D.C.: Noninvasive quantification of solid tumor microstructure using VERDICT MRI. Cancer Res. 74, 1902-1912 (2014)

8. Hall, M.G., Alexander, D.C.: Convergence and parameter choice for Monte-Carlo simulations of diffusion MRI. IEEE Trans. Med. Imaging 28, 1354-1364 (2009)

9. Yeh, C.H., Schmitt, B., Le Bihan, D., Li-Schlittgen, J.R., Lin, C.P., Poupon, C.: Diffusion microscopist simulator: a general Monte Carlo simulation system for diffusion magnetic resonance imaging. PLoS ONE 8, e76626 (2013)

10. Fieremans, E., De Deene, Y., Delputte, S., Özdemir, M.S., Achten, E., Lemahieu, I.: The design of anisotropic diffusion phantoms for the validation of diffusion weighted magnetic resonance imaging. Phys. Med. Biol. 53, 5405-5419 (2008)

11. Hubbard, P.L., Zhou, F.L., Eichhorn, S.J., Parker, G.J.M.: Biomimetic phantom for the validation of diffusion magnetic resonance imaging. Magn. Reson. Med. 73, 299-305 (2015)

12. Siow, B., Drobnjak, I., Chatterjee, A., Lythgoe, M.F., Alexander, D.C.: Estimation of pore size in a microstructure phantom using the optimised gradient waveform diffusion weighted NMR sequence. J. Magn. Reson. 214, 51-60 (2012)

13. Dietrich, O., Hubert, A., Heiland, S.: Imaging cell size and permeability in biological tissue using the diffusion-time dependence of the apparent diffusion coefficient. Phys. Med. Biol. 59, 3081-3096 (2014)

14. Zhou, F.L., Hubbard, P.L., Eichhorn, S.J., Parker, G.J.M.: Jet deposition in nearfield electrospinning of patterned polycaprolactone and sugar-polycaprolactone core-shell fibres. Polymer 52, 3603-3610 (2011)

15. Zhou, F.L., Hubbard, P.L., Eichhorn, S.J., Parker, G.J.M.: Coaxially electrospun axon-mimicking fibers for diffusion magnetic resonance imaging. ACS Appl. Mater. Interfaces 4, 6311-6316 (2012)

16. Malyarenko, D., Galbán, C.J., Londy, F.J., Meyer, C.R., Johnson, T.D., Rehemtulla, A., Ross, B.D., Chenevert, T.L.: Multi-system repeatability and reproducibility of apparent diffusion coefficient measurement using an ice-water phantom. J. Magn. Reson. Imaging 37, 1238-1246 (2013)

17. Zhang, L., Huang, J., Si, T., Xu, R.X.: Coaxial electrospray of microparticles and nanoparticles for biomedical applications. Expert Rev. Med. Devices 9, 595-612 (2012)

18. Murday, J.S., Cotts, R.M.: Self-diffusion coefficient of liquid lithium. J. Chem. Phys. 48, 4938-4945 (1968)

19. Neuman, C.H.: Spin echo of spins diffusing in a bounded medium. J. Chem. Phys. 60, 4508-4511 (1974)

20. Price, W.S., Barzykin, A.V., Hayamizu, K., Tachiya, M.: A model for diffusive transport through a spherical interface probed by pulsed-field gradient NMR. Biophys. J. 74, 2259-2271 (1998)

21. Bland, J.M., Altman, D.G.: Measuring agreement in method comparison studies. Stat. Methods Med. Res. 8, 135-160 (1999) 
22. Walker-Samuel, S., Orton, M., McPhail, L.D., Robinson, S.P.: Robust estimation of the apparent diffusion coefficient (ADC) in heterogeneous solid tumors. Magn. Reson. Med. 62, 420-429 (2009)

23. Kristoffersen, A.: Optimal estimation of the diffusion coefficient from non-averaged and averaged noisy magnitude data. J. Magn. Reson. 187, 293-305 (2007)

24. Yao, J., Lim, L.K., Xie, J., Hua, J., Wang, C.H.: Characterization of electrospraying process for polymeric particle fabrication. J. Aerosol Sci. 39, 987-1002 (2008) 